\title{
Numerical Simulation on the Effect of Turbulence Models on Impingement Cooling of Double Chamber Model
}

\author{
Zhenglei Yu, Tao Xu, Junlou Li, Hang Xiu, and Yun Li \\ College of Mechanical Science \& Engineering, Jilin University, Changchun 130025, China \\ Correspondence should be addressed to Yun Li; yunjlu@sina.com
}

Received 5 August 2013; Revised 17 October 2013; Accepted 17 October 2013

Academic Editor: Waqar Khan

Copyright (C) 2013 Zhenglei Yu et al. This is an open access article distributed under the Creative Commons Attribution License, which permits unrestricted use, distribution, and reproduction in any medium, provided the original work is properly cited.

\begin{abstract}
Investigation of the effects of impingement cooling for the different turbulence models and study of the aerodynamic behavior of a simplified transition piece model (TP) are the two themes of this paper. A model (double chamber model) of a one-fourth cylinder is designed which could simulate the transition piece structure and performance. The relative strengths and drawbacks of renormalization group theory $k-\varepsilon(\mathrm{RNG})$, the realizable $k-\varepsilon$ (RKE), the $v^{2}-f$, the shear stress transport $k-\omega$ (SST), and large-eddy simulation (LES) models are used to solve the closure problem. The prediction of the inner wall temperature, cooling effectiveness, and velocity magnitude contours in various conditions are compared in five different turbulence models. Surprisingly, the $v^{2}-f$ and SST models can produce even better predictions of fluid properties in impinging jet flows. It is recommended as the best compromise between solution speed and accuracy.
\end{abstract}

\section{Introduction}

Given the large number of sustained operational hours required for industrial turbines, two important demands placed on such engines are component life and overall engine performance. These demands are somewhat conflicting because high temperatures are required at turbine combustor in order to achieve high performance; however, increasing combustor outlet temperature in turn reduced component life, high repair costs, and downtime costs. Impingement cooling is an enhanced heat transfer method capable of cooling a transition piece (TP) without injecting cool air directly into the gas chamber. Cooling the transition piece from the gas inlet enables engineers to dissipate the heat load and maintains more uniform temperatures in the turbine region needed for efficient turbine [1].

In the example of turbine cooling applications [2], impinging jet flows may be used to cool several different sections of the engine such as the combustor case (combustor can walls), turbine case/liner, and the critical high temperature turbine blades. General applications and performance of impinging jets had been discussed in a number of reviews [3-6]. The jet impingement angle has an effect on heat transfer and was studied frequently [3, 4]. Göppert et al. [5] investigated the effects of an unstable precessing jet over a fixed target plate. Hwang et al. [6] altered the flow pattern in the initial shearing layer by using coaxial jets.

Numerical modeling of impinging jet flows and heat transfer is employed widely for prediction, sensitivity analysis, and device design. Finite element, finite difference, and finite volume computational fluid dynamics (CFD) models of impinging jets have succeeded in making rough predictions of heat transfer coefficients and velocity fields. Turbulent impinging jet CFD employs practically all available numerical methods that will be critically reviewed in the following sections.

An earlier critical review of this topic was conducted by Polat et al. [7] in 1989. Since that date, the variety of numerical models have been established and computational research in predicting the physical behavior of impinging jets. Tzeng et al. [8] compared seven low Re modifications of the $k-\varepsilon$ model using a confined turbulent slot jet array problem with three adjacent jets at $H / B=1$. Heck et al. [9] showed the RNG model that provided a close match of $\mathrm{Nu}$ in the wall-jet region but an error up to $10 \%$ in the stagnation region. Modeling by Cziesla et al. [10] demonstrated the ability of LES to predict local $\mathrm{Nu}$ under a slot jet within $10 \%$ of experimental measurements. Silieti et al. [11] investigated the numerical 
prediction of cooling effectiveness in $2 \mathrm{D}$ and $3 \mathrm{D}$ gas turbine end walls/shrouds for the cases of conjugate and adiabatic heat transfer models. They considered different cooling hole geometries, that is, cooling slots, cylindrical, and fan-shaped cooling holes at different blowing ratios. They incorporated the effects of different turbulence models in predicting the surface temperature and hence the cooling effectiveness.

There is a great interest in the application of impingement cooling to protect the transition piece from high temperature gas streams. The model created in the paper looks like a quarter torus with the curved double chambers simulating the structure of the transition piece. The relative strengths and drawbacks of renormalization group theory $k-\varepsilon$ model (RNG), the realizable $k-\varepsilon$ model (RKE), the $v^{2}-f$ model, the shear stress transport $k-\omega$ model (SST), and large-eddy simulation model (LES) for impinging jet flow and heat transfer are compared. As well, the velocity and temperature fields, in addition to centerline and two-dimensional impingement cooling effectiveness, will be presented.

\section{Turbulence Models}

2.1. The Renormalization Group Theory $k-\varepsilon$ Model (RNG). The RNG $k-\varepsilon$ turbulence model is derived from the instantaneous Navier-Stokes equations, using a mathematical technique called renormalization group (RNG) methods, borrowed from quantum mechanics. The analytical derivation results in a model with constants different from those in the standard $k-\varepsilon$ model, and it results in additional terms and functions in the transport equations for the turbulent kinetic energy $(k)$ and for dissipation rate $(\varepsilon)$. A more comprehensive description of RNG theory and its application to turbulence can be found in $[12,13]$. The governing equations for this model are as follows:

$k$ equation

$$
\begin{aligned}
\rho \frac{D k}{D t}= & \frac{\partial}{\partial x_{j}}\left[\left(\mu+\frac{\mu_{t}}{\sigma_{k}}\right) \frac{\partial k}{\partial x_{j}}\right]+\mu_{t} S^{2} \\
& -\rho \varepsilon-g_{i} \frac{\mu_{t}}{\rho \operatorname{Pr}_{k}} \frac{\partial \rho}{\partial x_{i}}-2 \rho \varepsilon \frac{k}{\gamma R T}
\end{aligned}
$$

$\varepsilon$ equation

$$
\rho \frac{D \varepsilon}{D t}=\frac{\partial}{\partial x_{j}}\left[\left(\alpha_{\tau} \mu_{\mathrm{eff}}\right) \frac{\partial \varepsilon}{\partial x_{j}}\right]+\frac{\varepsilon}{k}\left(C_{1 \tau} \mu_{\tau} S^{2}-\rho \varepsilon C_{2 \tau}^{*}\right),
$$

where $D / D t$ is the convective (or substantial time) derivative,

$$
\frac{D}{D t}=\frac{\partial}{\partial t}+\vec{V} \cdot \nabla
$$

and $S$ is related to the mean strain tensor, $S_{i j}=(1 / 2)\left(\partial u_{i} / x_{j}+\right.$ $\left.\partial u_{j} / x_{i}\right)$, as $S=\sqrt{2 S_{i j} S_{j i}}, C_{1 \tau}=1.42, C_{2 \tau}=1.68, C_{\mu}=$ $0.0845, \eta=S(k / \varepsilon), \mu_{t}=\rho C_{\mu}\left(k^{2} / \varepsilon\right)$, and $C_{2 \tau}^{*}=C_{2 \tau}+$ $\left(\left(C_{\mu} \rho \eta^{3}\left(1-\left(\eta_{1} / \eta_{0}\right)\right)\right) /\left(1+\beta \eta^{3}\right)\right)$.

2.2. The Realizable $k-\varepsilon$ Model (RKE). The term realizable means that the model satisfies certain mathematical constraints on the normal stresses consistent with the physics of turbulent flows. In this model, the $k$ equation is the same as in RNG model; however $C_{\mu}$ is not a constant and varies as a function of mean velocity field and turbulence (0.09 in log-layer $S(k / \varepsilon)=3.3,0.05$ in shear layer of $S(k / \varepsilon)=6$ ). The equation is based on a transport equation for the meansquare vorticity fluctuation [14] as

$$
\rho \frac{D \varepsilon}{D t}=\frac{\partial}{\partial x_{j}}\left[\left(\mu+\frac{\mu_{t}}{\sigma}\right) \frac{\partial \varepsilon}{\partial x_{j}}\right]+C_{1} S \rho \varepsilon-C_{2} \frac{\rho \varepsilon^{2}}{k+\sqrt{v e}},
$$

where $C_{1}=\max [0.43, \eta /(\eta+1)]$ and $C_{2}=1.0$. This model is designed to avoid unphysical solutions in the flow field.

2.3. The $v^{2}-f$ Model. Durbin's $v^{2}-f$ model, also known as the "normal velocity relaxation model," has shown some of the best predictions to date, with calculated $\mathrm{Nu}$ values falling within the spread of experimental data [15]. The $v^{2}-$ $f$ model uses an eddy viscosity to increase stability with two additional differential equations beyond those of the $k-\varepsilon$ model, forming a four-equation model. The additional equations are defined as

$$
\begin{aligned}
& \frac{D k}{D t}=\frac{\partial}{\partial x_{j}}\left[\left(v+v^{\prime}\right) \frac{\partial k}{\partial x_{j}}\right]+2 v^{\prime} S_{i j} S_{i j}-\varepsilon, \\
& v^{\prime}=C_{\mu} \overline{v^{2}} T_{\text {scale }}, \\
& \frac{D \varepsilon}{D t}=\frac{c_{\varepsilon 1}^{\prime} 2 v^{\prime} S_{i j} S_{i j}-c_{\varepsilon 2} \varepsilon}{T_{\text {scale }}}+\frac{\partial}{\partial x_{j}}\left[\left(v+\frac{v^{\prime}}{\sigma_{\varepsilon}}\right) \frac{\partial \varepsilon}{\partial x_{j}}\right], \\
& \frac{D \overline{v^{2}}}{D t}=k f_{\text {wall }}-\overline{v^{2}} \frac{\varepsilon}{k}+\frac{\partial}{\partial x_{j}}\left[\left(v+v^{\prime}\right) \frac{\partial \overline{v^{2}}}{\partial x_{j}}\right] \text {, } \\
& f_{\text {wall }}-L_{\text {scale }}^{2} \frac{\partial}{\partial x_{j}} \frac{\partial f}{\partial x_{j}} \\
& =\frac{\left(C_{1}-1\right)\left((2 / 3)-\left(\overline{v^{2}} / k\right)\right)}{T_{\text {scale }}}+\frac{C_{2} 2 v^{\prime} S_{i j} S_{i j}}{k}, \\
& L_{\text {scale }}=C_{L} \max \left(\min \left(\frac{k^{3 / 2}}{\varepsilon}, \frac{1}{\sqrt{3}} \frac{k^{3 / 2}}{\overline{v^{2}} C_{\mu} \sqrt{2 S_{i j} S_{i j}}}\right)\right. \text {, } \\
& \left.C_{\mu}\left(\frac{v^{3}}{\varepsilon}\right)^{1 / 4}\right) \\
& T_{\text {scale }}=\min \left(\max \left(\frac{k}{\varepsilon}, 6 \sqrt{\frac{\bar{v}}{\varepsilon}}\right), \frac{\alpha}{\sqrt{3}} \frac{k}{\overline{v^{2}} C_{\mu} \sqrt{2 S_{i j} S_{i j}}}\right), \\
& c_{\varepsilon 1}^{\prime}=1.44\left(1+0.045 \sqrt{\frac{k}{\overline{v^{2}}}}\right),
\end{aligned}
$$

where $C_{\mu}=0.19, c_{\varepsilon 2}=1.9, \sigma_{\varepsilon}=1.3, C_{1}=1.4, C_{2}=0.3$, $C_{\eta}=70.0, C_{L}=0.3$, and $\alpha=0.6$. Similar equations exist for predicting the transport of a scalar (e.g., thermal energy) with a $\operatorname{Pr}^{\prime}$ as a funtion of $v$ and $v^{\prime}$. 


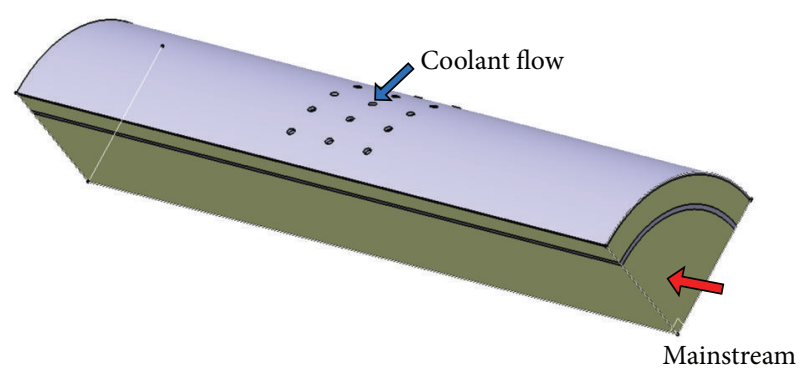

FIGURE 1: Impingement-cooling concave model.

2.4. The Shear Stress Transport $k-\omega$ Model (SST). There are two major ways in which the SST model differs from the standard $k-\omega$ model. The first is the gradual change from the standard $k-\omega$ model in the inner region of the boundary layer to a high Reynolds-number version of the $k-\varepsilon$ model in the outer part of the boundary layer. The second is the modified turbulent viscosity formulation to account for the transport effects of the principal turbulent shear stress. The SST (shear stress transport) model consists of the zonal (blended) $k-$ $\omega / k-\varepsilon$ equations and clips of turbulent viscosity so that turbulent stress stays within what is dictated by structural similarity constant. The $k$ equation is the same as the standard $k-\omega$ model whereas the resulting blended equation for $\omega$ is [16]

$$
\begin{aligned}
\rho \frac{D \omega}{D t}= & \frac{\gamma}{v_{t}} \tau_{i j} \frac{\partial U_{i}}{\partial x_{j}}-\rho \beta \omega^{2}+\frac{\partial}{\partial x_{j}}\left[\left(\mu+\frac{\mu_{\tau}}{\sigma_{\omega}}\right) \frac{\partial \omega}{\partial x_{j}}\right] \\
& +2 \rho\left(1-F_{1}\right) \sigma_{\omega 2} \frac{1}{\omega} \frac{\partial k}{\partial x_{j}} \frac{\partial \omega}{\partial x_{j}}
\end{aligned}
$$

where $F_{1}=1$ in the inner layer and $F_{1} \rightarrow 0$ in the outer layer, and $\sigma_{\omega 2}=1.168$.

2.5. Large-Eddy Simulation (LES). Large-eddy simulation (LES) is a technique intermediate between the direct simulation of turbulent flows and the solution of the Reynoldsaveraged equations. In LES the contribution of the large, energy-carrying structures to momentum and energy transfer is computed exactly; well only the effect of the smallest scales of turbulence is modeled. Since the small scales tend to be more homogeneous and universal and less affected by the boundary conditions than the large ones, there is hope that their models can be simpler and require fewer adjustments when applied to different flows than similar models for the RANS equations $[17,18]$.

\section{Computational Model, Boundary Conditions, and Grid}

3.1. Model Description. Transition piece develops heat transformation on both internal and external walls to eliminate resonant frequency concerns. As well the transition piece conducts gas flow directly from the corresponding combustion liners toward the first stage of the gas turbine

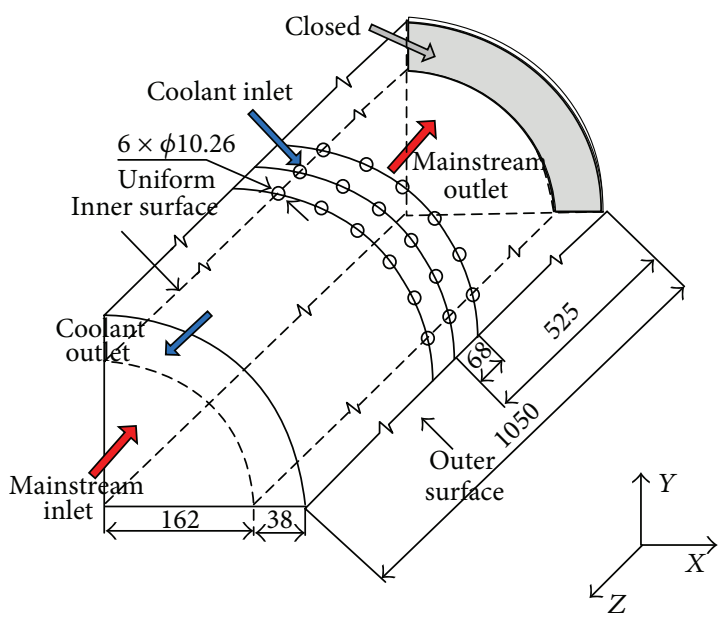

(a)

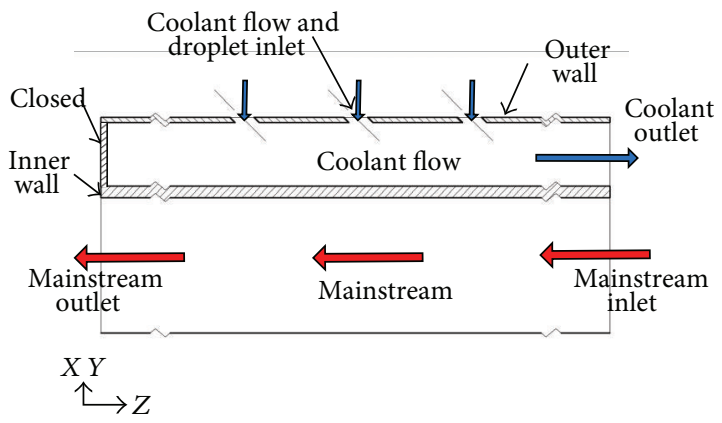

(b)

FIGURE 2: Computational domain showing boundary conditions.

(stator). The discrete coolant jets, forming a protective film chamber on the side of transition piece, are drawn from the upstream compressor in an operational gas turbine engine. From the supply plenum, the coolant is ejected through the several rows of discrete holes over the external boundary layer against the local high thermal conduction on the other side of the transition pieces [19]. The model, as a onefourth cylinder, could simulate the transition piece's structure and performance, to elaborate the turbulence effect on the impingement-cooling performance over a concave surface (see Figure 1) [4].

A schematic diagram of the flow domain along with boundary conditions and dimensions is given in Figure 2(a). As shown in the figure, the one-fourth cylinder model has two layers of chambers with a length of $1050 \mathrm{~mm}$ and an outer radius and an inner radius of $200 \mathrm{~mm}$ and $162 \mathrm{~mm}$, respectively. In the diagram, one side of the outer chamber called the coolant chamber is closed; contrarily, both sides of the mainstream chamber as the inner chamber are unfolding in which gas could flow through from one side to the other. There are 18 holes distributed uniformly in three rows on the surface of the outer wall. The distance between the two rows is $68 \mathrm{~mm}$ and the diameter of all the holes is about $10.26 \mathrm{~mm}$. In this scenario, the velocity of the coolant flow is set at $6 \mathrm{~m} / \mathrm{s}$; the temperatures of the coolant flow and the mainstream flow are set as $300 \mathrm{~K}$ and $1300 \mathrm{~K}$, respectively. 
TABLE 1: Boundary conditions.

\begin{tabular}{llc}
\hline Component & Boundary conditions & Magnitude \\
\hline \multirow{3}{*}{ Mainstream inlet } & Mass flux rate & $31.46(\mathrm{~kg} / \mathrm{s})$ \\
& Gas temperature & $1300(\mathrm{~K})$ \\
& Turbulent intensity & $5(\%)$ \\
& Hydraulic diameter & $0.324(\mathrm{~m})$ \\
& Pressure & $1.512(\mathrm{MPa})$ \\
Mainstream outlet & Turbulent intensity & $5(\%)$ \\
& Hydraulic diameter & $0.324(\mathrm{~m})$ \\
& Convection coefficient & $10\left(\mathrm{~W} / \mathrm{m}^{2} \mathrm{~K}\right)$ \\
& Air temperature & $300(\mathrm{~K})$ \\
& Pressure & $1.4552(\mathrm{MPa})$ \\
Coolant chamber & Pressure recovery coefficient & 0.95 \\
& Turbulent intensity & $5(\%)$ \\
& Hydraulic diameter & $0.01026(\mathrm{~m})$ \\
\hline
\end{tabular}

3.2. Boundary Conditions. Boundary conditions are applied to specific faces within the domain to specify the flow and thermal variables that dictate conditions within the model. Figure 2(a) discloses the boundary conditions used in the model in which the cooling air and gas are moving along, respectively, in the two layers of chambers in opposite directions. In the cooling chamber, the simulation is performed by using air as the cooling flow, while velocity and temperature are set on the jet holes, with pressure on the exit. In another chamber, it is assumed that the mainstream is a mixture of $\mathrm{O}_{2}, \mathrm{H}_{2} \mathrm{O}, \mathrm{CO}_{2}, \mathrm{~N}_{2}$, and some rare gases. The model created is considered as boundary condition (Table 1) [4]. Assumption of the solid wall of the quarter torus is formed with a hypothesis of negligible thermal resistance by conduction; the thermal properties of the material were considered by Nimonic 263.

3.3. Meshing and Simulation Procedures. The computational domain incorporates the model, the HEXA mesh in the software, ICEM/CFD, used to generate the structured multiblock and the body-fitted grid system. In this study, the grid system associated with the parts of the mainstream and the coolant supply plenum is H-type. Figure 3 shows the grids of the computational domain, and the total number of the cells for the $3 \mathrm{D}$ domain is 776,828 . Local grid refinement is used near the hole regions.

The wall $Y$-plus (7) is a nondimensional number similar to local Reynolds number, determining whether the influences in the wall-adjacent cells are laminar or turbulent, hence indicating the part of the turbulent boundary layer that they resolve:

$$
y^{+}=\frac{u_{\tau \text { air }} y}{v_{\text {air }}} .
$$

The subdivisions of the near-wall region in a turbulent boundary layer can be summarized as follows [20]:

(a) $y^{+}<5$ : in the viscous sublayer region (velocity profiles are assumed to be laminar and viscous stress dominates the wall shear),

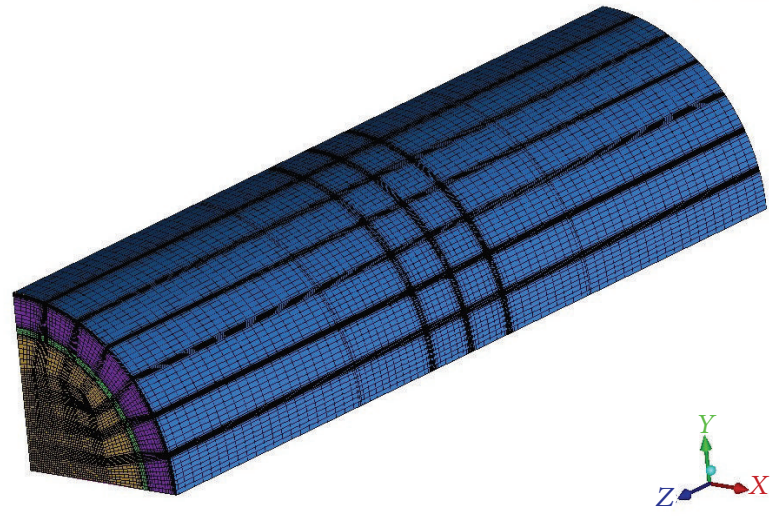

Figure 3: Meshes.

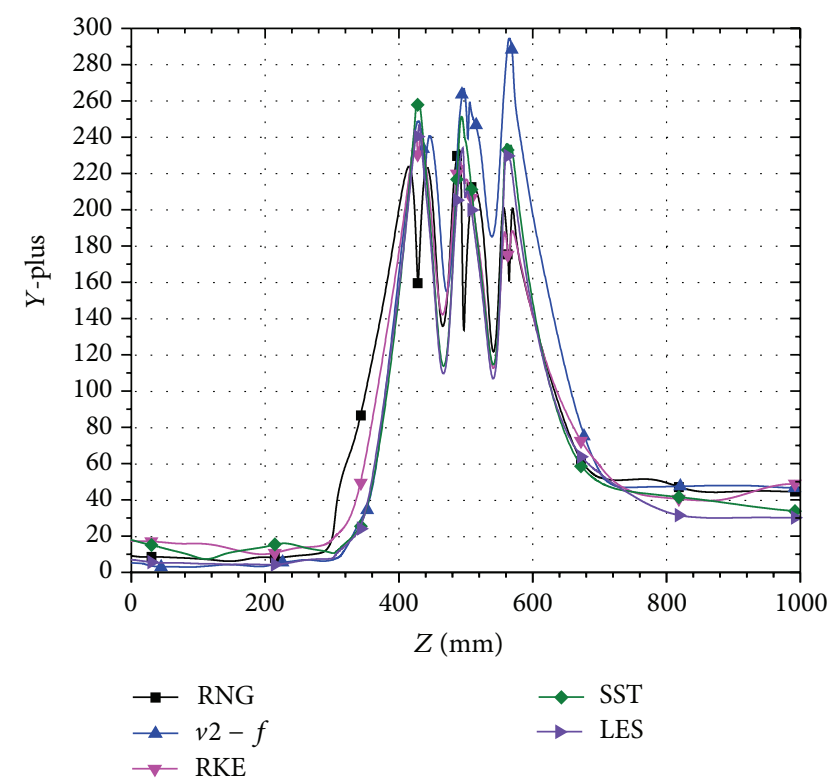

FIGURE 4: $Y$-plus distribution on the inner wall surface.

(b) $5<y^{+}<30$ : buffer region (both viscous and turbulent shear dominate),

(c) $30<y^{+}<300$ : fully turbulent portion or log-law region (corresponds to the region where turbulent shear predominates).

As it can be seen in Figure 4, for all cases, all nodes on the inner wall surface have the $Y$-plus value smaller than 300 .

This study is using a commercial CFD code based on the control-volume method, ANSYS-FLUENT 12.0.16, which in order to predict temperature, impingement-cooling effectiveness, and velocity fields. All runs were made on a PC cluster with sixteen Pentium-4 $3.0 \mathrm{GHz}$ personal computers. The convergence criteria of the steady-state solution are judged by the reduction in the mass residual by a factor of 6 , typically, in 2000 iterations. 


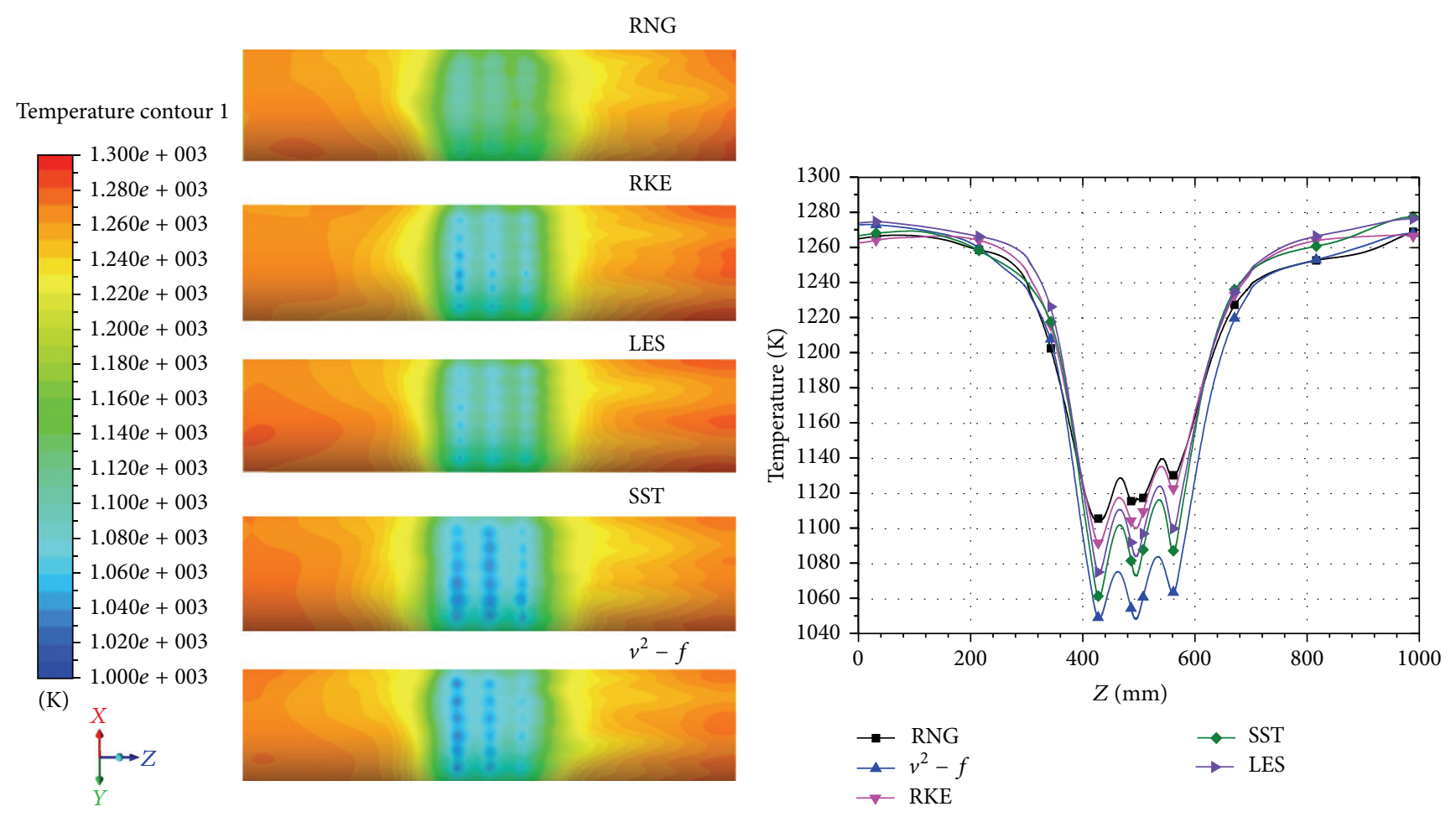

(a)

(b)

FIGURE 5: Comparative analysis of inner wall temperature using different turbulence models: (a) temperature distribution of inner wall; (b) temperature along the center line.

\section{Results and Discussion}

4.1. Inner Wall Temperature. Figure 5 shows the temperature contours predicted by five turbulence models. The figure illustrates that the temperature at the starting point of the wall is high and then starts to go down. The starting point is cooled by the coolant holes at the curve $Z=430 \mathrm{~mm}$ on the outer wall while the same temperature is maintained throughout the coolant holes. After the coolant strikes the inner wall, there are vortexes formed. The jet impingement and the vortex formed out of the coolant flow cool the surface of the inner wall. The delicate color region (orange, yellow, and green region) is approximately the region where the coolant strikes the inner wall after being reflected by the coolant holes, in which the inner wall is cooled purely by impingement cooling. These figures confirm that the $v^{2}-f$ model has well simulation of heat transfer in the double chamber model. The calculated temperature at the center line of the inner wall is presented in Figure 5(b); there is a difference among the predictions of SST, LES, and RKE models. Both the temperature contours and the color lines have the same trend in the five turbulence models whereas the calculated temperatures between $Z=400$ and $600 \mathrm{~mm}$ are diverse for all turbulence models. All turbulence models continue to predict similar wall temperatures, while the RNG model underpredicts the temperature by up to $5 \%-8 \%$ in the same location.
4.2. Cooling Effectiveness. To define cooling effectiveness, the surface temperature downstream of the cooling hole has to be measured. The adiabatic cooling effectiveness $(\eta)$ is used to examine the performance of cooling. The definition of $\eta$ is

$$
\eta=\frac{T_{m}-T_{\mathrm{aw}}}{T_{m}-T_{c}},
$$

where $T_{m}$ is the mainstream hot gas inlet temperature, which is a fixed value for calculation of the adiabatic cooling effectiveness of any location, and $T_{c}$ is the temperature of the coolant, which is assigned as a constant of $300 \mathrm{~K}$ in this issue. $T_{\mathrm{aw}}$ is the adiabatic wall temperature [21].

Figures 6(a) and 6(b) exhibit the comparison of cooling effectiveness on distribution and averaged cooling effectiveness along the $z$-axis, respectively. It could be concluded that the effectiveness is high at the beginning of the film while decreasing gradually in downstream. The distribution of the cooling effectiveness of the $v^{2}-f$ and SST model cases is significantly different. The centerline effectiveness by using the five turbulence model cases is shown in Figure 6(b). Overall, particularly the $v^{2}-f$ model gave better results compared to other models. Values of cooling effectiveness compare with the RNG model case, there is an approximately $30 \%$ difference in $v^{2}-f$ model case. 


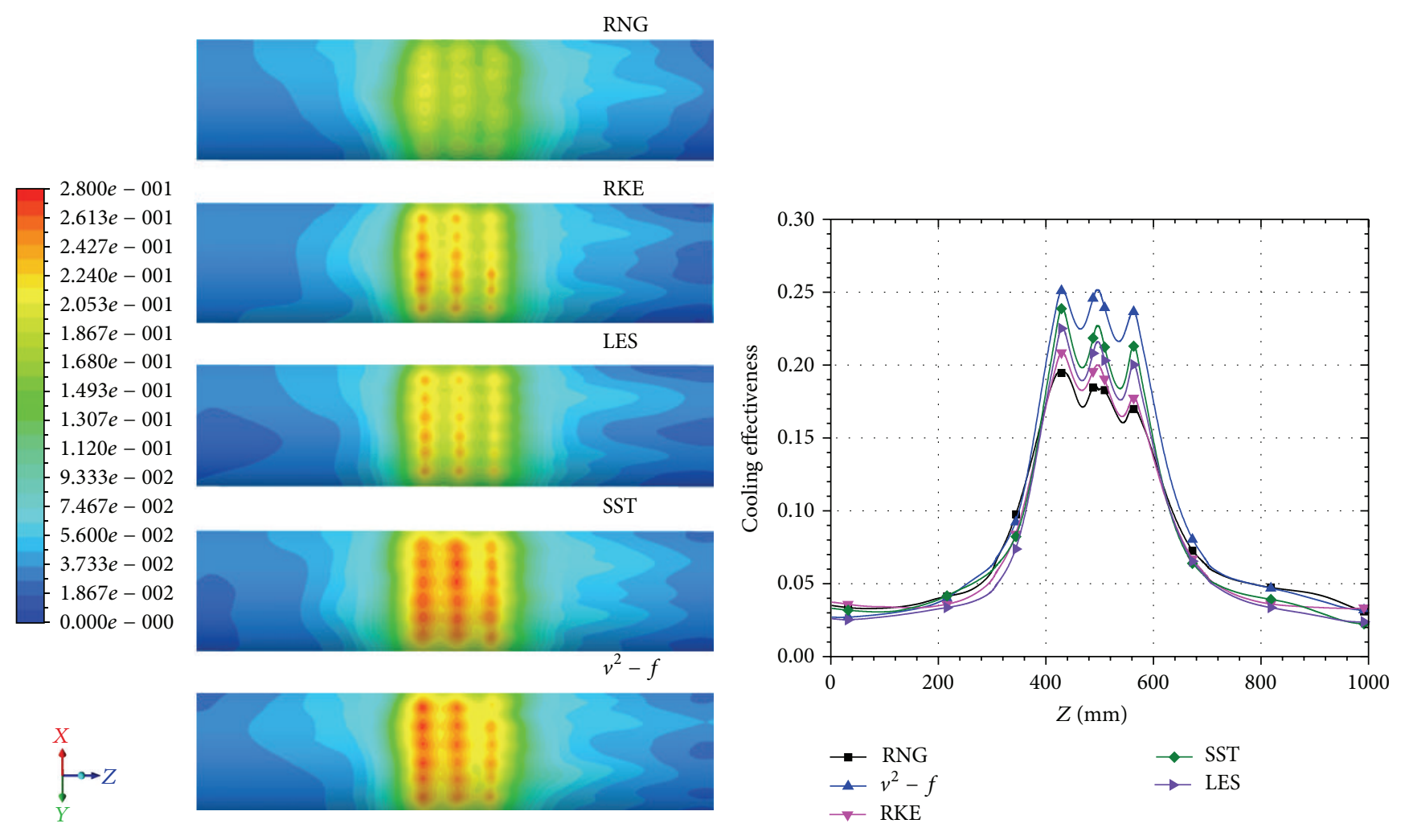

(a)

(b)

FIGURE 6: Comparative analysis of cooling effectiveness in five turbulence models: (a) cooling effectiveness distribution of inner wall; (b) averaged cooling effectiveness.

4.3. Characteristics of the Flow Field. Since the thermal field of a jet-in-crossflow interaction is dictated by the hydrodynamics, the flow field results were predicted by five turbulence models. Figure 7(a) shows the velocity contours at the section plane of coolant chamber. As it can be seen, all models predicted the low momentum region along the downstream edge and the corresponding high momentum or jetting region along the upstream edge within the cooling holes. The predicted reattachment region by SST and $v^{2}-f$ model cases is approximately at the inlet of the cooling chamber, whereas LES, RKE, and RNG predict a larger separation bubble and the flow separates behind the cooling holes. The computed near inner wall velocity contours $(\mathrm{m} / \mathrm{s})$ along the centerline plane are shown in Figure 7(b), where the turbulence closure was simulated using the five different turbulence models. All predictions are extremely close to each other with a skewed upstream velocity profile.

The actual computational cost will of course vary with model complexity and computing power. With the parallel computing resources of a desktop computer available at the time of writing, six Pentium- 4 typically $3 \mathrm{GHz}$ processors, for a high-resolution two-dimensional problem, the steady time-averaged eddy viscosity models (RNG, RKE) will have computation times of a few hours (0.5-1.5). In comparison, the more complex SST and $v^{2}-f$ could take 2-4 hours depending on how smoothly the model converges. Based on recent work, unsteady LES models have computation times at least two orders of magnitude higher; a well-resolved threedimensional LES impinging jet model could take a day to provide a solution.

\section{Conclusion}

A numerical simulation has been performed to study the flow and heat transfer of impinging cooling on the double chamber model, and a comparative study, indicating the ability of five turbulence models, is presented. The research of turbulence model tasks is important to improve the design and resulting performance of impinging jets.

During this investigation, numerical simulation is impacted with five turbulence models, which has some practical value for real processing and guiding significance for theory. To date, the SST and $v^{2}-f$ models offer the best results for the least amount of computation time. It is very important to consider the effect of heat conduction within the metal on the predictions of an accurate surface temperature and hence impingement-cooling effectiveness. The validation of the present study has confirmed angle cases and will be employed in future studies of impingement-cooling parameters optimization. 


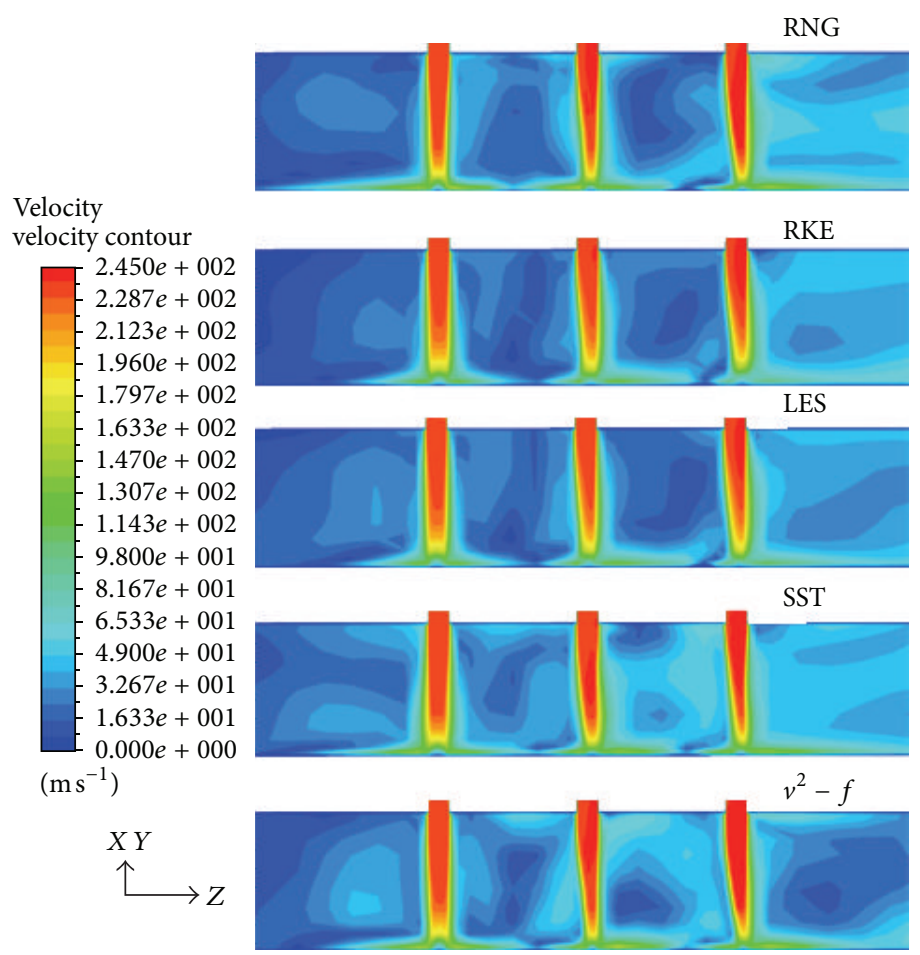

(a)

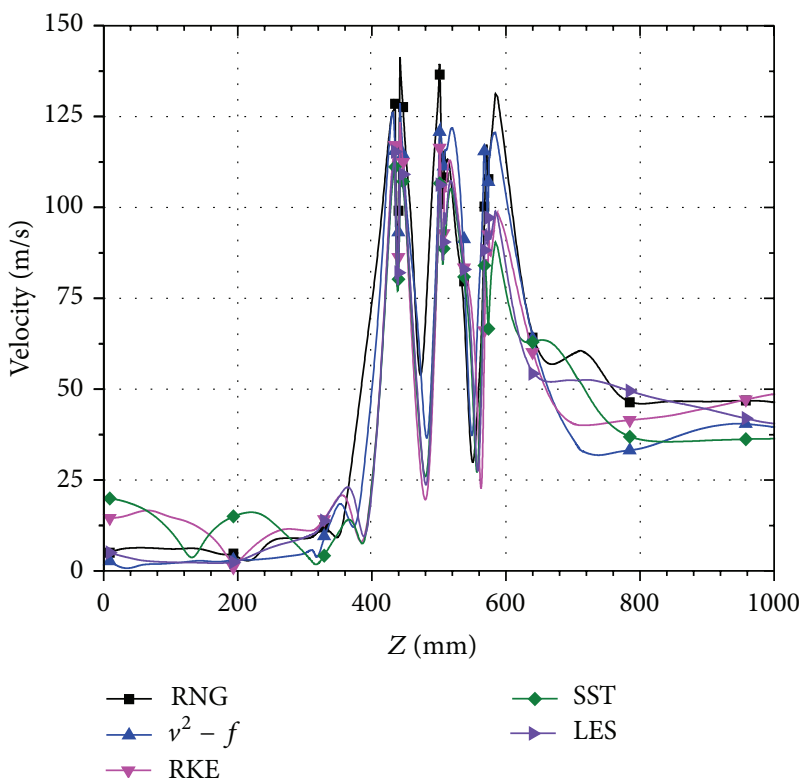

(b)

FIGURE 7: Coolant velocity contours predicted by various turbulence models: (a) $X-Y$ cross-section velocity distribution; (b) averaged coolant velocity.

\section{Nomenclature}

$D_{a}: \quad$ Diameter of coolant chamber

$D_{g}: \quad$ Diameter of mainstream chamber

$L: \quad$ Length of the model

$T: \quad$ Absolute static temperature

$X, Y, Z$ : Nondimensional coordinates in diameter, spanwise, and mainstream directions.

\section{Greek Symbols}

$\eta$ : Cooling effectiveness.

\section{Suffixes}

$m$ : Mainstream flow

$c$ : Coolant flow

aw: Adiabatic wall.

\section{Conflict of Interests}

The authors declare that there is no conflict of interests.

\section{Acknowledgment}

This research is supported by the Technology Development of Jilin Province (no. 20126001).

\section{References}

[1] J. C. Han, S. Dutta, and S. V. Ekkad, Gas Turbine Heat Transfer and Cooling Technology, Taylor and Francis, New York, NY, USA, 2000.

[2] N. Zuckerman and N. Lior, "Impingement heat transfer: correlations and numerical modeling," Journal of Heat Transfer, vol. 127, no. 5, pp. 544-552, 2005.

[3] A. A. Tawfek, "Heat transfer studies of the oblique impingement of round jets upon a curved surface," Heat and Mass Transfer, vol. 38, no. 6, pp. 467-475, 2002.

[4] Z. L. Yu, T. Xu, J. L. Li, L. Ma, and T. S. Xu, "Comparison of a series of double chamber model with various hole angles for enhancing cooling effectiveness," International Communications in Heat and Mass Transfer, vol. 44, pp. 38-44, 2013.

[5] S. Göppert, T. Gürtler, H. Mocikat, and H. Herwig, "Heat transfer under a precessing jet: effects of unsteady jet impingement," International Journal of Heat and Mass Transfer, vol. 47, no. 1213, pp. 2795-2806, 2004.

[6] S. D. Hwang, C. H. Lee, and H. H. Cho, "Heat transfer and flow structures in axisymmetric impinging jet controlled by vortex pairing," International Journal of Heat and Fluid Flow, vol. 22, no. 3, pp. 293-300, 2001.

[7] S. Polat, B. Huang, A. S. Mujumdar, and W. J. M. Douglas, "Numerical flow and heat transfer under impinging jets: a review," Annual Review of Numerical Fluid Mechanics and Heat Transfer, vol. 2, pp. 157-197, 1989.

[8] P. Y. Tzeng, C. Y. Soong, and C. D. Hsieh, "Numerical investigation of heat transfer under confined impinging turbulent slot jets," Numerical Heat Transfer A, vol. 35, no. 8, pp. 903-924, 1999. 
[9] U. Heck, U. Fritsching, and K. Bauckhage, "Fluid flow and heat transfer in gas jet quenching of a cylinder," International Journal of Numerical Methods for Heat and Fluid Flow, vol. 11, no. 1, pp. 36-49, 2001.

[10] T. Cziesla, G. Biswas, H. Chattopadhyay, and N. K. Mitra, "Large-eddy simulation of flow and heat transfer in an impinging slot jet," International Journal of Heat and Fluid Flow, vol. 22, no. 5, pp. 500-508, 2001.

[11] M. Silieti, E. Divo, and A. J. Kassab, "Film cooling effectiveness from a single scaled-up fan-shaped hole a CFD simulation of adiabatic and conjugate heat transfer models," in Proceedings of the ASME Turbo Expo: Power for Land, Sea, and Air, pp. 431-441, June 2005, paper no. GT2005-68431.

[12] S. H. Park, S. W. Park, S. H. Rhee, S. B. Lee, J.-E. Choi, and S. H. Kang, "Investigation on the wall function implementation for the prediction of ship resistance," International Journal of Naval Architecture and Ocean Engineering, vol. 5, no. 1, pp. 33-46, 2013.

[13] S.-E. Kim and S. H. Rhee, "Efficient engineering prediction of turbulentwing tip vortex flows," Computer Modeling in Engineering and Sciences, vol. 62, no. 3, pp. 291-309, 2010.

[14] Z. L. Yu, T. Xu, J. L. Li, T. S. Xu, and Y. Tatsuo, "Computational analysis of droplet mass and size effect on mist/air impingement cooling performance," Advances in Mechanical Engineering, vol. 2013, Article ID 181856, 8 pages, 2013.

[15] M. Behnia, S. Parneix, Y. Shabany, and P. A. Durbin, "Numerical study of turbulent heat transfer in confined and unconfined impinging jets," International Journal of Heat and Fluid Flow, vol. 20, no. 1, pp. 1-9, 1999.

[16] T. H. Park, H. G. Choi, J. Y. Yoo, and S. J. Kim, "Streamline upwind numerical simulation of two-dimensional confined impinging slot jets," International Journal of Heat and Mass Transfer, vol. 46, no. 2, pp. 251-262, 2003.

[17] Y. Huang, S. Wang, and V. Yang, "Systematic analysis of leanpremixed swirl-stabilized combustion," AIAA Journal, vol. 44, no. 4, pp. 724-740, 2006.

[18] V. Moureau, P. Domingo, and L. Vervisch, "From large-eddy simulation to direct numerical simulation of a lean premixed swirl flame: filtered laminar flame-PDF modeling," Combustion and Flame, vol. 158, no. 7, pp. 1340-1357, 2011.

[19] J. Benoit, C. Johnston, and M. Zingg, "Enhancing gas turbine power plant profitability: chronic transition piece and turbine part failures in some $501 \mathrm{~F}$ gas turbines led to a replacement part redesign," Power Engineering, vol. 111, no. 11, pp. 140-144, 2007.

[20] M. Moshfeghi, Y. J. Song, and Y. H. Xie, "Effects of near-wall grid spacing on SST-K- $\omega$ model using NREL Phase VI horizontal axis wind turbine," Journal of Wind Engineering and Industrial Aerodynamics, vol. 107, pp. 94-105, 2012.

[21] H. Wu, J. Wang, and Z. Tao, "Evaluation of predicted heat transfer on a transonic vane using $v^{2}-f$ turbulence models," Journal of Thermal Science and Technology, vol. 6, no. 3, pp. 424435, 2011. 


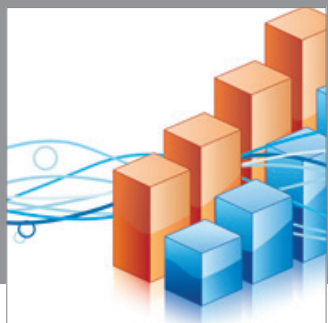

Advances in

Operations Research

mansans

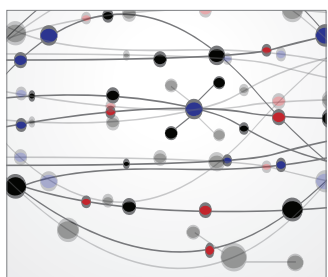

The Scientific World Journal
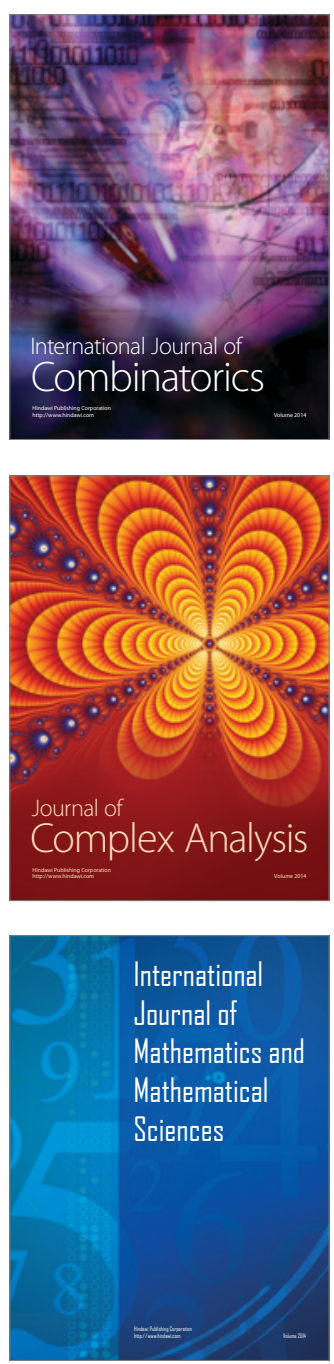
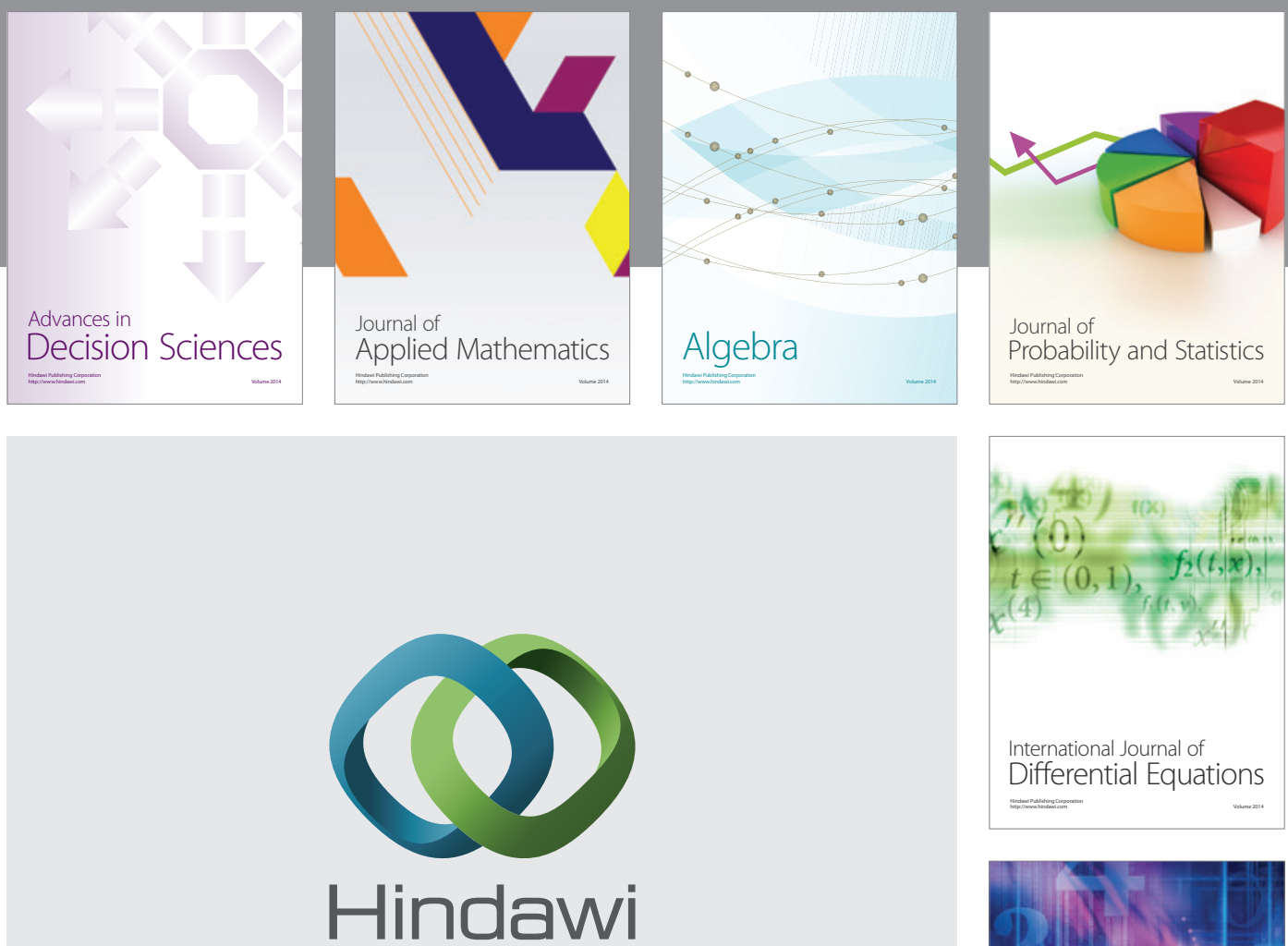

Submit your manuscripts at http://www.hindawi.com
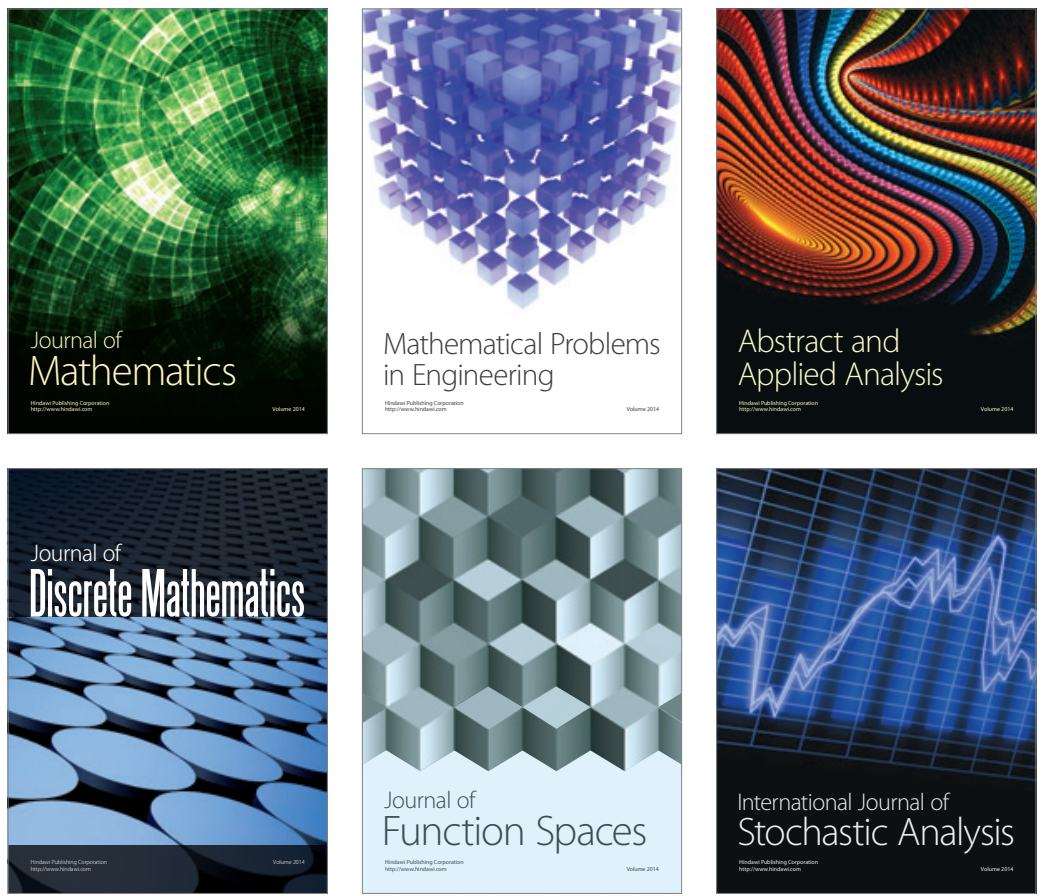

Journal of

Function Spaces

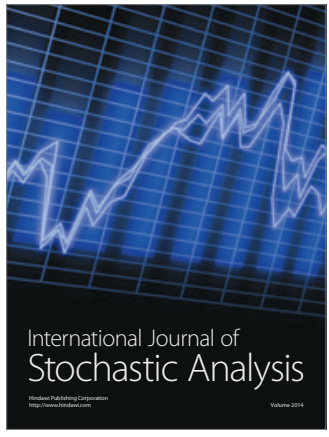

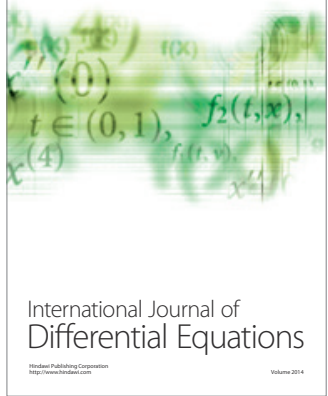
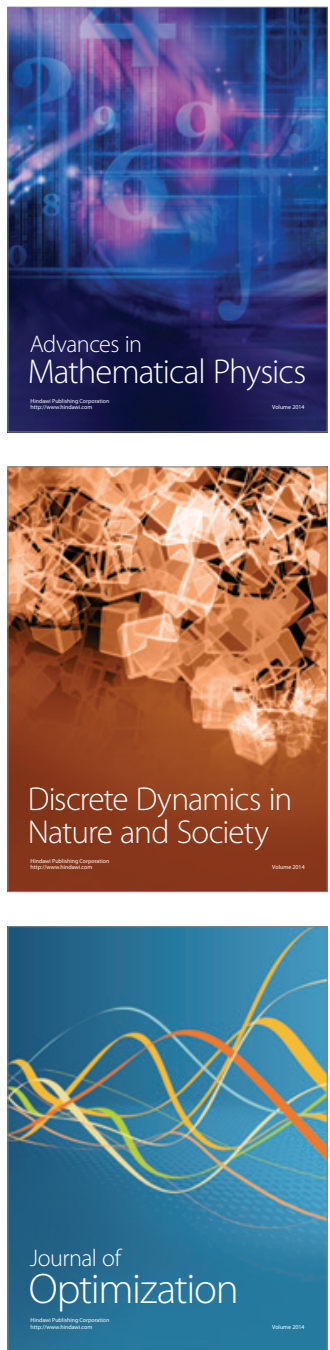